\title{
A simplified mathematical theory of MHD power generators
}

\begin{abstract}
We present a simplified version of the Faraday magnetohydrodynamics (MHD) generators theory. The effect of the Loretz force against the fluid flow is neglected, whence it follows the uniform flow of the plasma. We use Lazăr Dragoş's analytic solution for the electric potential and perform some numerical calculations in order to obtain the useful output power of a generator connected to an external resistive circuit.
\end{abstract}

\section{Adrian Carabineanu}

\section{Introduction}

In the last years, new applications of MHD generators to hypersonic aircrafts have been considered (see for example the papers of Petit and Geffray [2] and Sheikin and Kuranov [3]). The generated electricity can be used to power electromagnetic devices on board or to the so-called MHD bypass (i.e. MHD acceleration of the engine exhaust flow). The basic elements of a simple MHD generator (the so-called continuous electrode Faraday generator) are shown in figure 1.

In the domain bounded by the electrodes, a magnetic field of induction $\mathbf{B}_{0}$ is transversely applied to the motion of an electrically conducting fluid flowing with velocity $\mathbf{V}_{0}$ inside an insulated duct. Many papers are dedicated to studying the flow of electrically conducting fluids in a duct ( Carabineanu

Key Words: MHD generator, electric potential, conformal mapping, Voltera-Signorini problem, Gauss quadrature formulas, power.

2010 Mathematics Subject Classification: Primary 76W05; Secondary 31A25.

Received: December, 2014.

Revised: January, 2015

Accepted: February, 2015. 


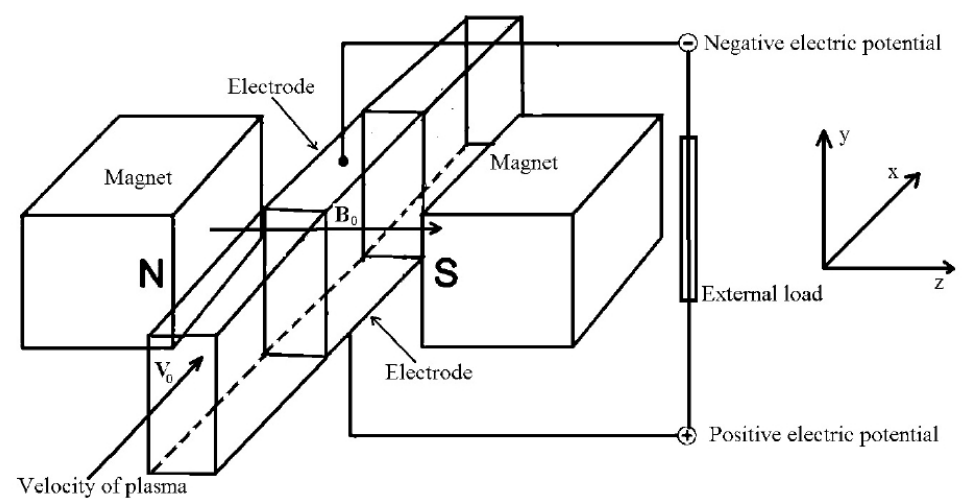

Figure 1: Faraday MHD generator

et al. [4], [5], Tezer-Sezgin [6], Bozkaya and Tezer-Sezgin, [7], Çelik [8], TezerSezgin and Han Aydin [9], etc.). References concerning the mathematical theory of the MHD power generators may be found in L. Dragoş's book [1], Chapter 4.

Electrically charged particles (ions and electrons) flowing with the fluid determine an induced electric field $\mathbf{V}_{0} \times \mathbf{B}_{0}$ which drives an electric current on a direction orthogonal to $\mathbf{V}_{0}$ and $\mathbf{B}_{0}$. The electric current is collected by the electrodes and flows in an external load circuit. Let $2 L_{0}$ be the distance between the electrodes. The electric current flowing across the electroconductive plasma between the electrodes is the Faraday current. It provides the main electrical output of the MHD power generator. The Faraday current reacts with the applied magnetic field creating a Hall effect current perpendicular to the Faraday current.

In this paper we present a simplified version of the MHD generator theory. Besides the simple geometry of the generator, we neglect the Hall effect and the effect of the Loretz force against the fluid flow, whence it follows the uniform flow of the plasma. Thermal effects, compressibility and viscosity are also neglected and the electromagnetic field is stationary. In order to calculate the MHD generator characteristics we use Lazăr Dragoş's analytic solution for the electric potential and perform some numerical calculations. A part of the article (Sections 1-5) was already presented in [12], where the values of the electric potential were imposed on the electrodes. Herein we consider a resistive external electric circuit and calculate the electric potential and the useful output power as functions of the resistence of the circuit. This is the original contribution of the present work. 


\section{Mathematical formulation of the problem}

We use dimensionless variables, by referring the electromagnetic field variables to $V_{0}, B_{0}$ and $L_{0}$. Denoting by $a$ the dimensionless length of the electrodes and by $\mathbf{i}, \mathbf{j}, \mathbf{k}$ the unit vectors of the Cartesian axes, in [1] one deduces that the dimensionless velocity and magnetic induction are:

$$
\mathbf{V}=\mathbf{i}, \mathbf{B}=\left\{\begin{array}{l}
\mathbf{k},|x| \leq a \\
\mathbf{0},|x|>a
\end{array}\right.
$$

Denoting by $\mathbf{J}$ the dimensionless current density and by $\mathbf{E}$ the dimensionless intensity of the electric field, we use Ohm's law

$$
\mathbf{J}=R m(\mathbf{E}+\mathbf{V} \times \mathbf{B}),
$$

where

$$
R m=\sigma \mu L_{0} V_{0}
$$

is the magnetic Reynolds number, $\sigma$ is the electrical conductivity and $\mu$ is the magnetic permeability.

From Faraday's law

$$
\nabla \times \mathbf{E}=\mathbf{0}
$$

we deduce that there exists a function $\varphi$ (the electric potential) such that

$$
\mathbf{E}=-\nabla \varphi
$$

From the jump condition

$$
[\mathbf{B}] \cdot \mathbf{n}=0
$$

and from the boundary conditions imposed on the insulating parts of the walls of the duct

$$
, \mathbf{J} \cdot \mathbf{n}=0, \mathbf{V} \cdot \mathbf{n}=0,
$$

we deduce that the flow is plane-parallel and the functions we are dealing with do not depend on the variable $z$.

In the sequel we shall calculate the potential of the electric field. From the continuity equation

$$
\nabla \cdot \mathbf{J}=0,
$$

from Ohm's law and from (5) it follows that

$$
\Delta \varphi=0,-\infty<x<\infty,-1<y<1 .
$$


Since the conductivity of electrodes is infinite, from Ohm's law we have $\frac{\partial \varphi(x, \pm 1)}{\partial x}=0$ and we may impose the following coditions

$$
\varphi(x, 1)=-\varphi_{w}, \varphi(x,-1)=\varphi_{w}, x \in(-a, a) .
$$

From Ohm's law, from (5) and from the boundary conditions (6), the following relationships

$$
\frac{\partial \varphi}{\partial y}(x, 1)=0, \frac{\partial \varphi}{\partial y}(x,-1)=0, x \in(-\infty,-a) \cup(a, \infty) .
$$

are deduced on the insulating parts of the walls of the duct.

At infinity one imposes the condition

$$
\lim _{|x| \rightarrow \infty} \mathbf{E}=-\lim _{|x| \rightarrow \infty} \nabla \varphi=0 .
$$

\section{Lazăr Dragoş's analytical solution [1]}

Since $\varphi(x, y)$ is a harmonic function, there exists its harmonic conjugate $\chi(x, y)$, related by $\varphi(x, y)$ through the Cauchy-Riemann equations

$$
\frac{\partial \varphi}{\partial x}=\frac{\partial \chi}{\partial y}, \frac{\partial \varphi}{\partial y}=-\frac{\partial \chi}{\partial x} .
$$

We shall also introduce the complex holomorphic function

$$
f(z)=\varphi(x, y)+i \chi(x, y), z=x+i y .
$$

From the boundary conditions (10), one deduces the boundary conditions

$$
\begin{gathered}
\chi(x, \pm 1)=b, x \in(-\infty,-a), \\
\chi(x, \pm 1)=b, x \in(a, \infty) .
\end{gathered}
$$

$\chi$ is determined up to an additive constant and $b$ has to be calculated.

With the conformal mapping

$$
\zeta=i \exp \frac{\pi}{2}(z+a), \zeta=\xi+i \eta,
$$

the strip $-1 \leq y \leq 1$ is mapped onto the upper half-plane $\eta \geq 0$ with the following point-to-point correspondence (figure 2):

$$
A(-\infty, \pm 1) \rightarrow A^{\prime}(0,0), B(-a,-1) \rightarrow B^{\prime}(1,0), C(a,-1) \rightarrow C^{\prime}(\exp \pi a, 0)
$$




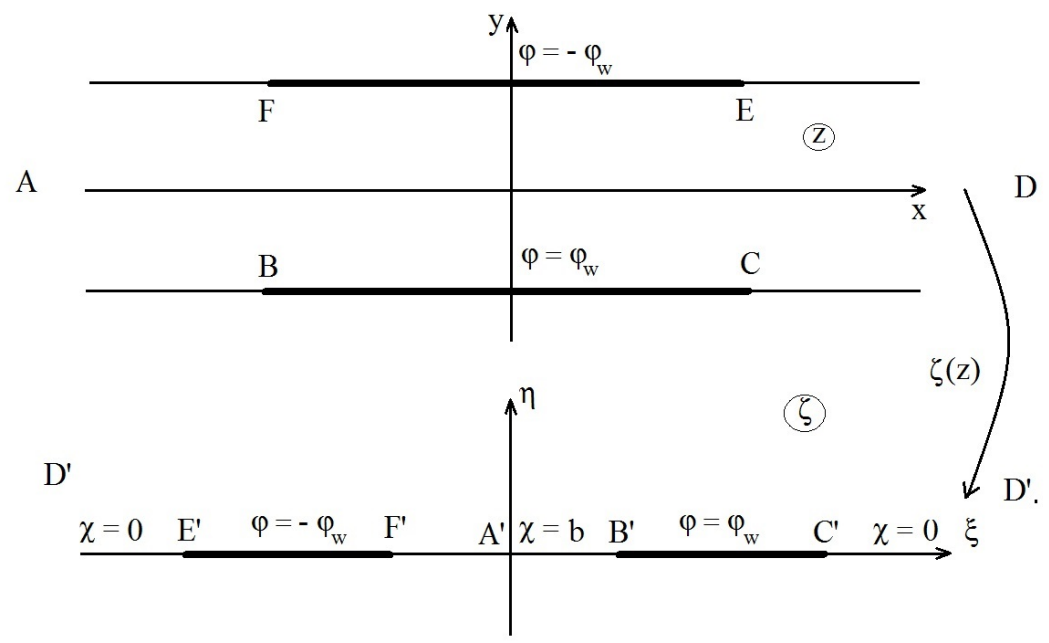

Figure 2: Conformal mapping and boundary correspondence

$D(\infty, \pm 1) \rightarrow D^{\prime}( \pm \infty, 0), E(a, 1) \rightarrow E^{\prime}(-\exp \pi a, 0), F(-a, 1) \rightarrow F^{\prime}(-1,0)$.

The boundary value problem (8) - (11) was reduced to the following Volterra-Signorini problem: find a holomorphic function $f(\zeta)=\varphi(\xi, \eta)+$ $i \chi(\xi, \eta)$ in the upper half-plane $\eta>0$, with the following boundary conditions:

$$
\begin{gathered}
\chi(\xi, 0)=0, \xi \in(-\infty,-\exp \pi a) \cup(\exp \pi a, \infty), \chi(\xi, 0)=b, \xi \in(-1,1) \\
\varphi(\xi, 0)=-\varphi_{w}, \xi \in(-\exp \pi a,-1), \varphi(\xi, 0)=\varphi_{w}, \xi \in(1, \exp \pi a) .
\end{gathered}
$$

The solution of the Volterra - Signorini problem, is given by a formula which may be found for example in [10], and it is ([1], Chapter 4):

$$
f(\zeta)=\frac{\sqrt{\left(\zeta^{2}-\exp 2 \pi a\right)\left(\zeta^{2}-1\right)}}{\pi} \int_{-\exp \pi a}^{\exp \pi a} \frac{\nu(\zeta)}{\sqrt{\left|\left(\xi^{2}-\exp 2 \pi a\right)\left(\xi^{2}-1\right)\right|}} \frac{d \xi}{\zeta-\xi}
$$

where

$$
\nu(\xi)=\left\{\begin{array}{c}
\varphi_{w}, \xi \in(-\exp \pi a,-1) \cup(1, \exp \pi a) \\
b, \xi \in(-1,1)
\end{array}\right.
$$

From condition (11) we deduce that $\lim _{\zeta \rightarrow \infty} \frac{d f}{d \zeta}=0$, whence, taking into 
account (19) and (20) it follows that the constant $b$ must satisfy the equation

$$
b \int_{-1}^{1} \frac{d \xi}{\sqrt{\left(\xi^{2}-\exp 2 \pi a\right)\left(\xi^{2}-1\right)}}=-2 \varphi_{w} \int_{1}^{\exp \pi a} \frac{d \xi}{\sqrt{\left(\exp 2 \pi a-\xi^{2}\right)\left(\xi^{2}-1\right)}} .
$$

\section{Numerical results}

We use a Gaussian quadrature formula for continuous functions ([11], Appendix $\mathrm{F})$ :

$$
\int_{-1}^{1} \frac{F(x)}{\sqrt{1-x^{2}}} d x \simeq \frac{\pi}{n} \sum_{\alpha=1}^{n} F\left(x_{\alpha}\right), x_{\alpha}=\cos \frac{(2 \alpha-1) \pi}{2 n}, \alpha=1, \ldots, n .
$$

Hence

$$
b=-\varphi_{w} \beta(a), \beta(a)=2 \frac{I_{1}(a)}{I_{2}(a)}
$$

with

$$
I_{2}(a)=\frac{\pi}{n} \sum_{\alpha=1}^{n} \frac{1}{\sqrt{\exp 2 \pi a-x_{\alpha}^{2}}} .
$$

We shall also use the Gaussian quadrature formula in order to calculate the integral from the right hand part of (21). To this aim we consider the change of variable

$$
\xi=\frac{\exp \pi a-1}{2} \theta+\frac{\exp \pi a+1}{2}
$$

and obtain

$$
\int_{1}^{\exp \pi a} \frac{d \xi}{\sqrt{\left(\exp \pi a-\xi^{2}\right)\left(\xi^{2}-1\right)}}=
$$

$2 \int_{-1}^{1} \frac{1}{\sqrt{[(\exp \pi a-1) \theta+3 \exp \pi a+1][(\exp \pi a-1) \theta+3 \exp \pi a+3]}} \frac{d \theta}{\sqrt{1-\theta^{2}}}$

$$
\begin{gathered}
\simeq \frac{\pi}{n} \sum_{\alpha=1}^{n} \frac{1}{\sqrt{\left[(\exp \pi a-1) x_{\alpha}+3 \exp \pi a+1\right]\left[(\exp \pi a-1) x_{\alpha}+3 \exp \pi a+3\right]}} \\
=I_{1}(a) .
\end{gathered}
$$

At the points $\left(x_{p}, y_{s}\right)$ of a certain grid we calculate

$$
\frac{\partial \varphi\left(x_{p}, y_{s}\right)}{\partial x}-i \frac{\partial \varphi\left(x_{p}, y_{s}\right)}{\partial y}=\frac{\pi i}{2} \frac{d f\left(\zeta_{p s}\right)}{d \zeta} \cdot \exp \frac{\pi}{2}\left(z_{p s}+a\right)
$$




$$
z_{p s}=x_{p}+i y_{s}, \zeta_{p s}=\zeta\left(z_{p s}\right) .
$$

We take into account that

$$
\begin{gathered}
\frac{d f\left(\zeta_{p s}\right)}{d \zeta}= \\
\frac{\zeta_{p s}\left(2 \zeta_{p s}^{2}-1-\exp 2 \pi a\right)}{\pi \sqrt{\left(\zeta_{p s}^{2}-\exp 2 \pi a\right)\left(\zeta_{p s}^{2}-1\right)}} \int_{-\exp \pi a}^{\exp \pi a} \frac{\nu(\zeta)}{\sqrt{\left|\left(\xi^{2}-\exp 2 \pi a\right)\left(\xi^{2}-1\right)\right|}} \frac{d \xi}{\zeta_{p s}-\xi}- \\
-\frac{\sqrt{\left(\zeta_{p s}^{2}-\exp 2 \pi a\right)\left(\zeta_{p s}^{2}-1\right)}}{\pi} \int_{-\exp \pi a}^{\exp \pi a} \frac{\nu(\zeta)}{\sqrt{\left|\left(\xi^{2}-\exp 2 \pi a\right)\left(\xi^{2}-1\right)\right|}} \frac{d \xi}{\left(\zeta_{p s}-\xi\right)^{2}} .
\end{gathered}
$$

For calculating $\frac{d f\left(\zeta_{p s}\right)}{d \zeta}$ we have to numerically compute the integrals

$$
\begin{gathered}
\int_{-1}^{1} \frac{b}{\sqrt{\left(1-\xi^{2}\right)\left(\exp 2 \pi a-\xi^{2}\right)}} \frac{d \xi}{\zeta_{p s}-\xi}, \\
\int_{-1}^{1} \frac{b}{\sqrt{\left(1-\xi^{2}\right)\left(\exp 2 \pi a-\xi^{2}\right)}} \frac{d \xi}{\left(\zeta_{p s}-\xi\right)^{2}}, \\
\int_{1}^{\exp \pi a} \frac{\varphi_{w}}{\sqrt{\left(\xi^{2}-1\right)\left(\exp 2 \pi a-\xi^{2}\right)}} \frac{d \xi}{\zeta_{p s}-\xi}, \\
\int_{1}^{\exp \pi a} \frac{\varphi_{w}}{\sqrt{\left(\xi^{2}-1\right)\left(\exp 2 \pi a-\xi^{2}\right)}} \frac{d \xi}{\left(\zeta_{p s}-\xi\right)^{2}} \\
\int_{1}^{\exp \pi a} \frac{d \xi}{\sqrt{\left(\xi^{2}-1\right)\left(\exp 2 \pi a-\xi^{2}\right)}} \frac{\varphi_{w}}{\zeta_{p s}+\xi} \\
\int_{1}^{\exp \pi a} \frac{d \xi}{\sqrt{\left(\xi^{2}-1\right)\left(\exp 2 \pi a-\xi^{2}\right)}} \frac{\left.d \zeta_{p s}+\xi\right)^{2}}{\left(\zeta^{2}\right.}
\end{gathered}
$$

To calculate the integrals from (29) and (30) we use the Gaussian quadrature formula (22), while for (31), (32), (33) and (34) we first perform the change of variable (25) and then use the Gaussian quadrature formula (22).

We use (2), (5), (27) and (28) to calculate the current density at the grid points. In figure 3 we present the current density field for $a=1 / 2$ and $\varphi_{w}=1$. 


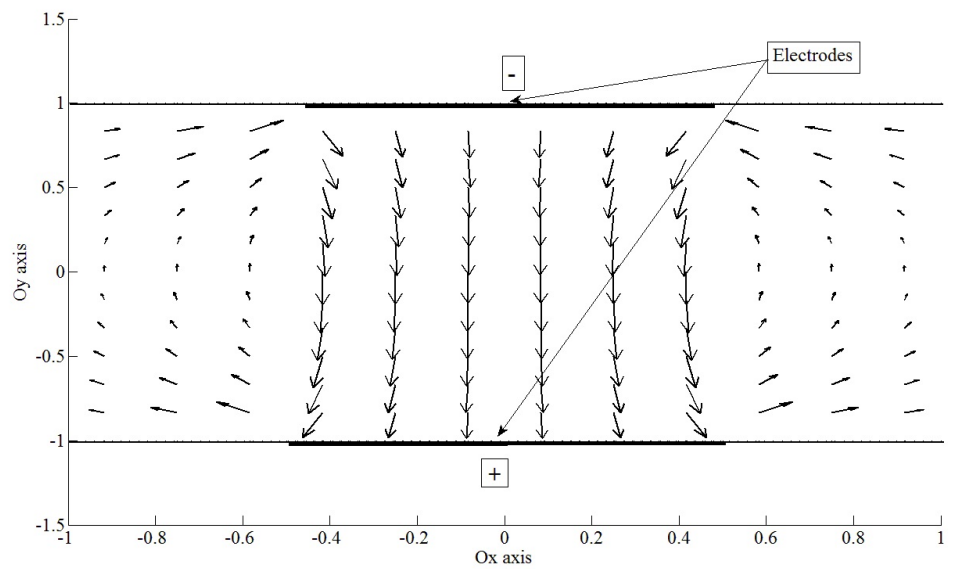

Figure 3: Current density field

\section{The characteristics of the MHD generator}

The dimensionless power on the unit of length developed by plasma in the motion against the electromagnetic field is

$A=-\iint_{(-\infty, \infty) \times[-1,1]} \mathbf{V} \cdot(\mathbf{J} \times \mathbf{B}) d x d y=\iint_{(-\infty, \infty) \times[-1,1]} \mathbf{J} \cdot(\mathbf{V} \times \mathbf{B}) d x d y$,

and it represents in fact the power dissipated by the Lorentz force with changed sign.

Taking into account the relations (1), (5), (9), (10) and Ohm's law (2) we get

$$
\begin{gathered}
A=R m \iint_{[-a, a] \times[-1,1]}\left(\frac{\partial \varphi}{\partial y}+1\right) d x d y= \\
R m \int_{-a}^{a}[\varphi(x, 1)-\varphi(x,-1)-2] d x=4 a R m\left(1-\varphi_{w}\right),
\end{gathered}
$$

and also

$$
A=\iint_{(-\infty, \infty) \times[-1,1]} \mathbf{J} \cdot\left(\frac{\mathbf{J}}{R m}-\mathbf{E}\right) d x d y=Q+W
$$


where

$$
Q=\iint_{(-\infty, \infty) \times[-1,1]} \frac{\mathbf{J}^{2}}{R m} d x d y>0
$$

stands for the Joule dissipation power and

$$
W=-\iint_{(-\infty, \infty) \times[-1,1]} \mathbf{E} \cdot \mathbf{J} d x d y
$$

is the useful output power of the generator.

From (5), from the continuity equation (7) and from the boundary conditions imposed on the insulating walls, one deduces that

$$
\begin{gathered}
W=\iint_{(-\infty, \infty) \times[-1,1]} \nabla \cdot(\varphi \mathbf{J}) d x d y=\int \varphi \mathbf{J} \cdot \mathbf{n} d s= \\
=-\varphi_{w} \int_{-a}^{a}[\mathbf{J}(x,-1)+\mathbf{J}(x, 1)] \cdot \mathbf{j} d x= \\
=\varphi_{w} R m \int_{-a}^{a}\left[\frac{\partial \varphi}{\partial y}(x,-1)+\frac{\partial \varphi}{\partial y}(x, 1)+2\right] d x= \\
=-\varphi_{w} R m \int_{-a}^{a}\left[\frac{\partial \chi}{\partial x}(x,-1)+\frac{\partial \varphi}{\partial x}(x, 1)-2\right] d x= \\
\varphi_{w} R m[\chi(-a .-1)-\chi(a,-1)+\chi(-a, 1)-\chi(a, 1)+2 a]= \\
=2 \varphi_{w} R m(2 a+b)=2 \varphi_{w} R m\left[2 a-\varphi_{w} \beta(a)\right] .
\end{gathered}
$$

\section{The calculus of the electric potential of the electrodes}

The electric current collected by the electrodes is used on the external circuit. The dimensionless intensity of this electric current is

$$
\begin{gathered}
I=-\mathbf{j} \cdot \int_{-a}^{a} \mathbf{J}(x,-1) d x=R m \int_{-a}^{a}\left[\frac{\partial \varphi}{\partial y}(x,-1)+1\right] d x= \\
2 a R m-R m \int_{-a}^{a} \frac{\partial \chi}{\partial x}(x,-1) d x=2 a-\chi(a,-1)+\chi(-a,-1)=\operatorname{Rm}(2 a+b) .
\end{gathered}
$$

We assume that the external electric circuit is a resistive circuit and we denote by $R$ its dimensionless equivalent resistance. According to Ohm's law we have

$$
2 \varphi_{w}=R I=R R m(2 a+b)=R R m\left(2 a-\varphi_{w} \beta(a)\right)
$$



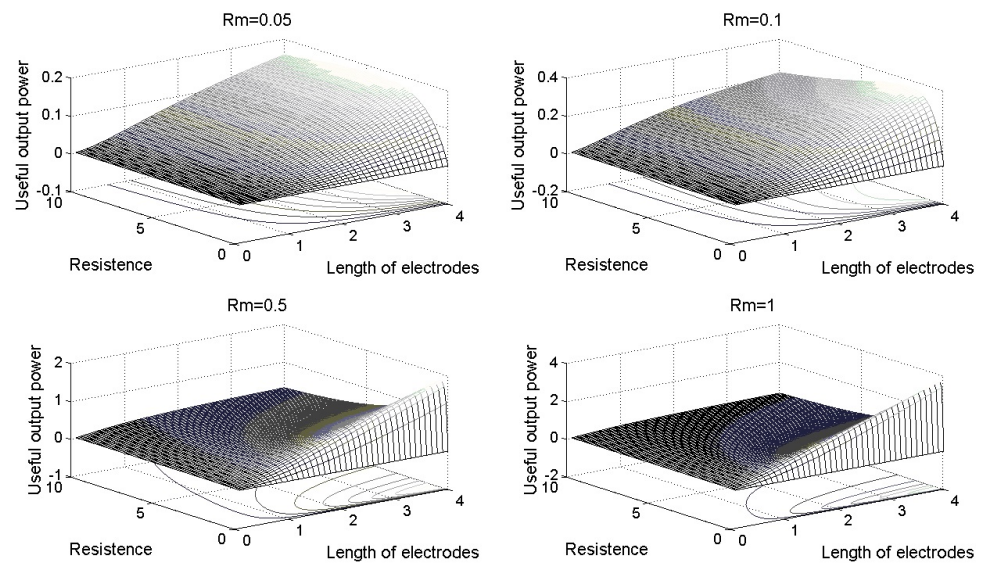

Figure 4: Useful output power

whence it follows

$$
\varphi_{w}=\frac{2 a R R m}{2+R R m \beta(a)} .
$$

From (39) and (42) we may calculate the dimensionless useful output power

$$
W=\frac{16 a^{2} R R m^{2}}{[2+R R m \beta(a)]^{2}} .
$$

In figure 4 we present the useful output power against the length of the electrodes and the resistence of the external circuit for various values of the magnetic Reynolds' number.

\section{References}

[1] L. Dragoş, Magnetofluid Dynamics, Ed. Academiei - Abacus Press, Bucureşti - Tunbridge Wells, Kent, ISBN 085626016 9, 1975.

[2] J. P. Petit, J. Geffray, MHD Flow - Control for Hypersonic Flight, Acta Physica Polonica A, vol. 115, issue 6, pp.1149-1151, 2009.

[3] A. L. Kuranov, E. G. Sheikin, Magnetohydrodynamic control on hypersonic aircraft under "Ajax" concept, J. Spacecraft and Rockets, ISSN: 0022-4650, EISSN: 1533-6794 vol. 40, no. 2, pp. 174-182, 2003. 
[4] A. Carabineanu, E. Lungu, Pseudospectral method for MHD pipe flow, Int J. Numer. Methods Engng., Online ISSN: 1097-0207, vol.68, issue 2, pp.173-191, 2006.

[5] A. Carabineanu, A. Dinu, I. Oprea, The application of the boundary element method to the magnetohydrodynamic duct flow, Z. Angew Math. Phys. ZAMP., Print ISSN 0044-2275, Online ISSN 1420-9039, vol. 46, issue 6, DOI: 10.1007/BF00917881, pp.971-981, 1995.

[6] M. Tezer-Sezgin, Solution of magnetohydrodynamic flow in a rectangular duct by differential quadrature method, Computers 83 Fluids, ISSN: 004579300, vol. 33, issue 4, DOI: 10.1016/S0045-7930(03)00072-, pp. 533-547, 2004.

[7] C. Bozkaya, M. Tezer-Sezgin, Solution of magnetohydrodynamic flow in a rectangular duct by differential quadrature method, Computers $\&$ Fluids, ISSN: 0045-7930, vol. 66, pp. 177-182, 2012.

[8] I. Çelik, Solution of magnetohydrodynamic flow in a rectangular duct by Chebyshev collocation method, Int. J. Numer. Meth. Fluids, Online ISSN: 1097-0363, vol. 66, issue 10, pp.1325-1340, 2011.

[9] S. Han Aydin, M. Tezer-Sezgin, A DRBEM solution for MHD pipe flow in a conducting medium, J. Comput. Appl. Math., ISSN:0377-0427, vol. 259, B, doi:10.1016/j.cam.2013.05.010, pp. 720-729, 2014.

[10] C. Jacob, Introduction mathématique à la mécanique des fluides, Editura Academiei - Gauthier Villars, Bucureşti, Paris, 1959.

[11] L. Dragos, Mathematical methods in aerodynamics, Kluwer, London Editura Academiei, Bucureşti, 2003.

[12] A. Carabineanu, Numerical Calculation of the Output Power of a MHD Generator, INCAS BULLETIN, Volume 6, Issue 4/ 2014, pp. 15 - 22 ISSN $2066-8201$.

Adrian CARABINEANU,

Department of Mathematics,

University of Bucharest,

Str. Academiei 14, 010014 Bucureşti, Romania.

"G. Mihoc-C.Iacob" Institute of Mathematical Statistics

and Applied Mathematics of Romanian Academy,

Calea 13 Septembrie 13, Bucureşti.

Email: acara@fmi.unibuc.ro 